\title{
Effectiveness of Integrated Approach of Yoga Therapy (IAYT) Versus Usual Care in Management on Chronic Low Back Pain Patients: a Randomized Controlled Pilot Study
}

\section{Spoorthi Poojari}

PGIMER

Deepanshu Dhiman

Government multispeciality hospital chandigarh sector 16

Babita Ghai ( $\square$ ghaibabita1@gmail.com )

PGIMER

\section{Deepali Mathur}

Apollo Hospitals

Kashinath Metri

Swami Vivekananda Yoga Anusandhana Samsthana

\section{Ketan Kataria}

Tata Memorial Hospital

\section{Akshay Anand}

PGIMER

\section{Research Article}

Keywords: Chronic low back pain, Integrated yoga therapy, TNF-a, Salivary CGRP, $\beta$-endorphins, Verbal numeric rating score, Modified Oswestery Disability Index

Posted Date: January 31st, 2022

DOl: https://doi.org/10.21203/rs.3.rs-1123520/v1

License: (c) (i) This work is licensed under a Creative Commons Attribution 4.0 International License. Read Full License 


\section{Abstract}

This is a controlled randomized trial which evaluated the clinical and molecular changes resulting from Integrated approach of yoga therapy (IAYT) as an adjunct regimen and compared it with usual care for the management of chronic low back pain patients. We enrolled 29 adult patients with chronic low back pain (CLBP). Patients were randomly divided into two groups. Control group received the usual care of treatment as per institutional protocol. The yoga group received IAYT as an adjunct to usual care. Primary outcomes were pain Intensity assessed by verbal numerical rating scale (VNRS) and functional ability assessed by Modified Oswestry Disability Index (MODI). Secondary outcomes were pain catastrophizing, quality of life, fear of movement related to CLBP, type of pain, levels of ß-Endorphin and TNF $a$ and Salivary CGRP. All parameters were measured at baseline, one month, and three months.

Significant decrease in VNRS score at 1 and 3 months was observed in both the groups with yoga group showing a more significant reduction in pain over time than the control group $(p=0.036)$. MODI improved significantly only in the yoga group at 1 and 3 months. Intergroup comparison revealed significantly better MODI over time in the yoga group ( $p<0.001$ ). DN4, PDQ, PCS, HADS (anxiety) and Euro QOL had a statistically significant improvement at 1 month and 3 months in the Yoga group compared to the Control group. The HADS (depression) had a statistically significant reduction scores in yoga group at 3 months compared to control group ( $p=0.012$ ). There was a significant reduction in TNF- $a$ values in the yoga group compared to baseline $(\mathrm{p}=0.004)$. IAYT therapy helped in addressing the psychological components of pain and improved quality of life patients with chronic low back pain compared to usual care.

\section{Introduction}

Low back pain (LBP) is the most common and potentially disabling public health problem worldwide. [1, 2] Globally, it is amongst the top four leading causes of Years lived with Disability (YLDs) as determined by the Global Burden of Disease 2017 study for 354 diseases across 195 countries. [3] Also the prevalence of LBP in India is alarming and nearly 60 percent of individuals suffer from it at some point during their lives.[3] The estimated lifetime, point and 1 year prevalence $(95 \% \mathrm{Cl})$ of LBP in India is reported to be $57 \%$ (54-59\%), 32\% (28-30\%), 48\% (46-51\%) and 59\% (56-62\%), respectively in a recent community-based study. In this community study women, increasing age and lifting activities were most significant positive predictors of LBP. [4]

Chronic low back pain (CLBP) is a much more complex problem affecting various aspects of an individual's life including biological, psychological, social, functional, and financial. LBP is reported to result in significant disability, which critically affects the quality of life of the patients. [5] A quarter of patients reported depression.[5] Despite various modalities options available for CLBP, management is usually suboptimal or inadequate. ${ }^{5}$ Most of these modalities seem to mainly focus on the biomedical aspect of pain and overlook the mind-body component.[6] Yoga, as an adjunct treatment for CLBP, has emerged as a potential intervention, supported by various randomized controlled trials,[7-9] systematic reviews,[10] meta-analysis, and clinical guidelines.[11] As CLBP patients are more susceptible to 
psychological and psychosocial factors, Mind-Body Stress Release (MBSR) offers a beneficial technique for back pain and has also been adopted by more than 18,000 Americans.[8, 12] Based on a similar approach of the MBSR technique, the Integrated Approach of Yoga Therapy (IAYT) can offer a perspective and synergistic culmination for effective management in CLBP.[13-15]

There is a dearth of data on evaluating IAYT in the Indian CLBP population despite the Indian origin of Yoga. Also, very few studies have examined the role of pain biomarkers like Brain-derived Neurotrophic factor (BDNF), C- Reactive Protein (CRP), and Tumor Necrosis Factor Alpha (TNF a) in evaluating the effects of yoga on CLBP patients. [16-18] To the best of our knowledge, no prior research has investigated the mechanistic role of Yoga on pain biomarkers with CLBP patients in the Indian population.

We, therefore, conducted a pilot study to evaluate the clinical and molecular changes resulting from IAYT administration as an adjunct regimen to the usual care for the management of CLBP patients.

\section{Material And Methods}

\section{Study design}

This is a randomized controlled trial employing a $2 \times 3$ repeated measure design with two groups and three assessment periods (pre-intervention, 1 month and 3 months follow up) conducted in the pain clinic of the Department of Anaesthesia and Intensive care of a tertiary care Institute. The study period was from 15th May2020 -15th August 2020.

\section{Ethics approval and trial registration}

The study was approved by the Institute Ethics Committee and collaborative Yoga University (SVYASA). The study was registered with the Clinical Trial Registry of India dated 30/04/2020 with CTRI registration number as: CTRI/2020/04/024951 http://ctri.nic.in/Clinicaltrials/pdf_generate.php?

trialid=42947\&EncHid=84466.70773\&modid=1\&compid=19\%27,\%2742947det\%27) and has adopted the CONSORT standard guidelines for clinical trial reporting.

\section{Enrolment}

All consecutive patients with CLBP attending the pain clinic were screened for the inclusion criteria. Inclusion criteria were patients with lumbosacral CLBP of more than 3 months duration not responding to conservative management with a verbal numerical rating scale of $\geq 5$ at the time of enrolment. The eligible adult CLBP patients were shortlisted at the pain clinic outpatient department (OPD). Based on the initial evaluation of physician for symptoms and radio-diagnostic methods, the selected patients were further approached by the researcher confirming on their diagnosis and pain status. The patients were given a detailed explanation regarding the study (potential risks/benefits, voluntary participation, procedures, etc.) and were provided with adequate time to reflect on the information. The enrolled 
patients were called in 4 batches, taking care of all the social distancing measures and COVID appropriate behavior.

\section{Diagnosis}

The assessment of patients was based on comprehensive clinical evaluation and assessing their symptoms, the degree of functional limitation, and psychological wellbeing. Radio-diagnostic methods involved MRI scans of the lumbosacral spine.

During the pre-treatment assessment, written informed consent was obtained from the patients. An initial pain history interview was conducted along with the administration of the baseline questionnaires.

\section{Randomization:}

The patients were randomly divided into the two groups. Control group received the usual care of treatment as per institutional protocol. The yoga group received IAYT as an adjunct to usual care.

The study used a block randomization design. A random allocation sequence was generated by the statistician. For each participant, an opaque envelope was opened, in consecutive order, by an external research assistant not involved in the inclusion process. The participants did not know the content of the different intervention arms. The yoga leader was not blinded. However, the research group assessing the study's outcome was blinded during the data collection and data analysis.

After randomization, the participants received information about the offered intervention from a research assistant not involved in the offered interventions. Yoga, strength training, and evidence-based advice were all presented as well-established interventions, to equalize the participants' expectations.

Participants then completed the 3-months treatment program that they were allotted into, while both the groups continued with their routine medical care. These patients were allowed to interact and communicate with the physician, the study investigator, and the therapist via telecommunication method whenever the need arise. Follow-up assessments were conducted in-person in four batches at one month and three months intervals post-treatment.

\section{STUDY INTERVENTION:}

\section{Yoga group}

The chosen IAYT protocol was designed for CLBP patients based on extensive research and contribution towards the promotion of health and efficiently treating pathologies for more than 30 years now. The protocol was planned with the inputs from the previous studies, yoga experts, expert medical professionals, physiotherapists and was further streamlined with the help of literature review, Yoga investigators, physiotherapists, and expert medical professionals. 
IAYT is a treatment protocol with a combination of modern scientific approaches blended with ancient wisdom, based on the basic principle of Panchkoshas, that is, five layers of existence to human beings. These practices were aimed at body level (Annamaya Kosa) include loosening stretches, asanas (postures); at subtle energy level (Pranamaya Kosa) including pranamaya (breathing practices), and the mind level (Manomaya Kosa) including meditation and relaxation techniques.[12]

The series of 60 minutes IAYT classes using all of the components of yoga (body, subtle energy, and mind level), was conducted for 2 weeks (6 sessions/week, 12 sessions total). The sessions were designed for participants to gradually progress from simple poses to more challenging poses adjusting to the patient's development. No props were used. Before the session, the participants were advised to keep their bowel and bladder empty. During the practice, care was taken regarding individual body limitations, and was advised not to strain too much to attain the correct posture with advice to suggest that.

It was followed by yoga practice at home and follow-ups at the set intervals for a total span of three months. Home practice for at least 45 mins was strongly encouraged throughout the intervention period after sessions ended. We provided the participants with a four-page all-inclusive colored IAYT pamphlet with pictorial presentations depicting practices as well as the audio recordings of the complete protocol to be used at home.

\section{Control group}

Patients in the control group were provided with usual care as per institutional protocol involving education about disease process and role of self-care, ergonomic and posture advice, advice to stay physically active, lifestyle modification, provision of a back care booklet (developed and validated at the part taking site) [19], medications as per the decision of treating physician, etc. They adhered to the treatment protocol for the same duration, under the guidance of a pain physician and physiotherapist.

\section{Participant Adherence}

Adherence to the yoga protocol was defined as attending at least 9 of the 12 yoga classes. For the usual care group, adherence was defined as reading at least three-fourths of the book by self-report.

The participants were assessed to adherence to home sessions by keeping a logbook of record the time spent on practicing and surprise video callings. A WhatsApp group was created for encouragement and motivation at regular intervals.

\section{Clinical Assessment and Follow up}

Baseline assessments included pain intensity using Verbal Numerical rating scale (VNRS)[20]functional ability using Modified Oswestry Disability Questionnaire (MODQ),[21]types of pain using Douleur neuropathic- 4 questionnaire (DN4)[22] and Pain Disability Questionnaire (PDQ),[23]pain catastrophizing using pain catastrophizing scale (PCS), [24]Quality of life using EuroQol (EQ-5D-5L) [24] 5patients mental and emotional state using Hospital Anxiety and Depression Scale (HADS) [26] and fear of movement related to CLBP using Tampa Scale of Kinesiophobia (TSK) [27].Patients were followed up at 
1 and 3 months and the abovementioned assessment were recorded. Medications prescribed by pain physician for CLBP were also assessed at all the three-time points. These drug therapies were reported as 'mono therapy' for 1 medication and 'dual therapy' for 2 medications. Further; medicine dosages were also evaluated to assess the changes in the overall dosage requirement. The patients rated their change on this 7-point scale that ranged from "very much improved" to "very much worse," with "no change" as the mid-point. $[28,29]$ The investigators recording data at baseline and follow-up were blinded to the treatment group.

Data collection on any unfavorable outcomes or serious health events was also collected.

\section{Biomarker assessment:}

We measured ß-Endorphin and TNF-a from the blood samples; and CGRP from the saliva of the CLBP patients to study the mechanistic role of yoga at baseline, one month and three months. ß-Endorphin is involved in pain management and is a chief natural suppressor of hyperexcitability.[30, 31]TNF- $a$ is an inflammatory marker that can interact with other neurotrophic factors and modify pain sensation. ${ }^{32}$ CGRP is involved in the transmission of pain and pain perception.[33, 34]

$5 \mathrm{ml}$ of blood was collected early morning (empty stomach) at baseline, 1 and 3 months. The samples were collected in a serum separator vacutainers kept at room temperature for half an hour followed by centrifugation at $2500 \mathrm{rpm}$ for 30 minutes. The serum was then separated, aliquoted, coded and subsequently stored at $-80^{\circ} \mathrm{C}$ until use.

For the saliva collection, the participants were instructed to rinse their mouth with distilled deionized water to remove debris and moisture, before the collection. Post 10 minutes of rest, the salivary collection process was commenced. During the collection, the participants were asked to keep their eyes open during salivation and not to speak or mentally stimulate salivary flow. The participants were instructed to sit upright and with their head slightly titled forward, non-stimulated whole saliva pooling at the floor of the mouth was gathered in a collection tube.

The expression level of $ß$-Endorphin, and TNF- $a$ in the serum and CGRP in the saliva was analyzed using commercially available ELISA kits (Qayee Bio, China). The OD was observed at 450nm on the ELISA reader.

\section{SAMPLE SIZE AND STATISTICAL ANALYSIS}

In the absence of available published data comparing IAYT with usual care in Indian CLBP patients for management as well as assessing the mechanistic molecular role of IAYT, we performed this pilot feasibility study. We performed post hoc power analysis to calculate the power of study. We conducted both intentions to treat (ITT) and per-protocol analysis with ITT being primary. Data of all patients having one follow-up at one month was included with the last observation carried forward imputation for missing data. 
Descriptive statistics including means, medians, and standard deviations for continuous variables, and the number and proportions for categorical variables are reported. Data are presented as mean $\pm S D$ and median (IQR) and were analyzed for normality using Kolmogorov-Smirnov Z test. Between-group (Yoga Group vs Control Group) comparisons of normally distributed variables were performed using an independent t-test; while the Wilcoxon-Mann-Whitney test was used for non-normally distributed variables. Categorical data were analyzed by $\chi 2$ test or Fischer exact test where appropriate. Generalized estimating equations (GEE) was used for the analysis of repeatedly measured data at a various time interval (NRS, MODI, DN4, PDQ, PCS, EuroQOL, HADS, and TSK) using linear regression to estimate mean change from baseline. GEE was used to include all available data, and account for intra-subject correlations on repeated measurements, and explore the overall average effect. For GEE, all observed data was used without imputation for missing values. The analysis was considered to be unbiased under a missing at-random (MAR) assumption. To account for correlation among repeated measures for the same individual, an unstructured covariance for the initial model was assumed. A simpler model was used for the covariance if appropriate. Results are presented as between-group differences in adjusted mean with 95\% confidence intervals (Cl) of the differences and effect sizes (ES) (adjusted mean difference/pooled baseline SD). The analysis was carried at a $5 \%$ level of significance level $(a=0.05)$. SPSS software version 22 (Chicago, Illinois: SPSS Inc.) was used for data analysis.

\section{Results}

Of the 87 patients screened for eligibility, 65 were found eligible. A total of 35 patients were approached for enrolment after fulfilling the inclusion criteria. Out of these 35 patients, four patients did not turn up for the initial assessment. The final sample consisted of 31 enrolled participants $(n=14$ Yoga Group and $\mathrm{n}=17$ Control group). 29 patients completed the assessment at the three time points (preintervention, 1 month \& 3month) and 2 patients were lost to follow up from the control group at one month $(n=15)$. The flow of participants is presented in the CONSORT flow diagram [Figure 1]

\section{Baseline demographic data:}

The baseline socio-demographic characteristics (age, sex, weight, height, and occupation) were comparable in both the Control and Yoga group [Table 1]. 
Table 1

Baseline socio-demographic data. **Significant at $p<0.05,1$ : Wilcoxon-Mann-Whitney U Test, 2: Fisher's Exact Test, 3: Chi-

Squared Test, values are represented as mean \pm SD and number (percentage).

\begin{tabular}{|c|c|c|c|}
\hline \multirow[t]{2}{*}{ Parameters } & \multicolumn{2}{|l|}{ Group } & \multirow[t]{2}{*}{$\mathrm{p}$ value } \\
\hline & $\begin{array}{l}\text { Yoga } \\
(n=14)\end{array}$ & $\begin{array}{l}\text { Control } \\
(n=15)\end{array}$ & \\
\hline Age (Years) & $36.93 \pm 10.34$ & $34.27 \pm 8.86$ & $0.346^{1}$ \\
\hline Age Group & & & $0.837^{2}$ \\
\hline 18-30 Years & $3(21.4 \%)$ & $5(33.3 \%)$ & \\
\hline 31-40 Years & $6(42.9 \%)$ & $7(46.7 \%)$ & \\
\hline 41-50 Years & $4(28.6 \%)$ & $3(20.0 \%)$ & \\
\hline 51-60 Years & $1(7.1 \%)$ & $0(0.0 \%)$ & \\
\hline Gender & & & $0.812^{3}$ \\
\hline Male & $5(35.7 \%)$ & $6(40.0 \%)$ & \\
\hline Female & $9(64.3 \%)$ & $9(60.0 \%)$ & \\
\hline Weight (Kg) & $66.50 \pm 14.63$ & $65.73 \pm 9.89$ & $0.878^{1}$ \\
\hline Height (cm) & $160.68 \pm 13.50$ & $166.13 \pm 5.28$ & $0.093^{1}$ \\
\hline Occupation & & & $0.271^{2}$ \\
\hline Housewife & $5(35.7 \%)$ & $8(53.3 \%)$ & \\
\hline Others & $8(57.1 \%)$ & $4(26.7 \%)$ & \\
\hline Farmer & $0(0.0 \%)$ & $2(13.3 \%)$ & \\
\hline Student & $1(7.1 \%)$ & $1(6.7 \%)$ & \\
\hline
\end{tabular}

\section{Baseline clinical variables:}

The baseline clinical variables (duration of symptoms, diagnosis, drug therapy, drug dosage, VNRS, MODQ, PDQ, DN4, EuroQOL, PCS, HADS, TSK, and biomarkers were comparable in both the Control and Yoga group. [Table 2] 
Table 2

Baseline clinical variables. Significant at $p<0.05$, 1: Wilcoxon-Mann-Whitney U Test, 2: Fisher's Exact Test, 3: Chi-Squared Test, values represented as mean $\pm S D$, number (percentages)

\begin{tabular}{|c|c|c|c|}
\hline Parameter & $\begin{array}{l}\text { Yoga group } \\
(n=14)\end{array}$ & $\begin{array}{l}\text { Control group } \\
(n=15)\end{array}$ & $\begin{array}{l}p \\
\text { value }\end{array}$ \\
\hline Duration Of Symptoms (Months) & $41.86 \pm 76.62$ & $41.13 \pm 42.52$ & $0.208^{1}$ \\
\hline Diagnosis & & & $0.890^{2}$ \\
\hline B/L Legs Pain & $6(42.9 \%)$ & $6(40.0 \%)$ & \\
\hline Right/Left Leg Pain & $5(35.7 \%)$ & $7(46.7 \%)$ & \\
\hline LBA & $3(21.4 \%)$ & $2(13.3 \%)$ & \\
\hline Drug Therapy (Baseline) & & & $0.356^{3}$ \\
\hline Monotherapy & $6(42.9 \%)$ & $9(60.0 \%)$ & \\
\hline Dual Therapy & $8(57.1 \%)$ & $6(40.0 \%)$ & \\
\hline Pregabalin Dose (mg/Day) (Baseline) & $78.57 \pm 67.12$ & $85.00 \pm 66.68$ & $0.836^{1}$ \\
\hline Amitriptyline Dose (mg/Day) (Baseline) & $9.29 \pm 2.67$ & $8.33 \pm 6.45$ & $0.403^{1}$ \\
\hline $\begin{array}{l}\text { Pregabalin+Amitriptyline Dose (mg/Day) } \\
\text { (Baseline) }\end{array}$ & $87.86 \pm 66.35$ & $93.33 \pm 64.13$ & $0.875^{1}$ \\
\hline VNRS (Baseline)*** & $7.57 \pm 0.58$ & $7.47 \pm 1.13$ & $0.77^{1}$ \\
\hline MODQ (Baseline) & $49.86 \pm 16.33$ & $50.93 \pm 10.14$ & $0.913^{1}$ \\
\hline PDQ (Baseline) & $9.79 \pm 5.95$ & $10.40 \pm 5.37$ & $0.930^{1}$ \\
\hline DN4 (Baseline) & $2.79 \pm 1.93$ & $3.33 \pm 1.91$ & $0.536^{1}$ \\
\hline EuroQOL (Baseline) & $0.22 \pm 0.27$ & $0.35 \pm 0.19$ & $0.162^{1}$ \\
\hline PCS (Baseline) & $34.43 \pm 10.26$ & $29.60 \pm 7.76$ & $0.143^{1}$ \\
\hline HAS (Depression) (Baseline) & $8.64 \pm 4.40$ & $7.47 \pm 2.77$ & $0.234^{1}$ \\
\hline HADS (Anxiety) (Baseline) & $11.07 \pm 4.43$ & $10.60 \pm 3.70$ & $0.709^{1}$ \\
\hline TSK (Baseline) & $42.64 \pm 5.20$ & $45.8 \pm 4.81$ & $0.090^{1}$ \\
\hline
\end{tabular}

\section{Primary outcome:}


Primary outcomes of the study were VNRS and MODQ at 3 months. The VNRS improved significantly over time in both the groups compared to baseline, (yoga group $p<0.001$, control group $p<0.001$ ). The reduction in VNRS in the yoga group was significantly more in comparison to the control group over time $(p=0.036$; using GEE). At 1 month the mean difference between the two groups was 1.35 (95\% Cl:0.15 to $2.55)$ and the effect size was $0.83(p=0.04)$. At 3 months the mean difference was 1.13 ( $95 \% \mathrm{Cl}: 0.61$ to $2.87)$ and the effect size was $0.48(p=0.21)$. [Figure 2]

MODI improved significantly in the Yoga group over time $(\mathrm{p}=0.049)$, however, no significant improvement was observed in the Control group ( $p=0.949)$. Between-group comparison revealed significantly better MODI over time in the Yoga group compared to the control group (GEE; $p<0.001$ ). At 1 month the mean difference between the two groups was 13.81 (95\% Cl:6.04 to 21.58) and the effect size was 1.33 $(p=0.002)$. At 3 months the mean difference was 17.62 (95\% Cl:7.83 to 27.41$)$ and the effect size was $1.35(p=0.002)$. [Figure 3]

\section{Secondary outcomes:}

DN4, PDQ, PCSand HADS (anxiety) had a statistically significant reduction at 1 month and 3 months in the Yoga group compared to the Control group [Table 3]. The HADS (depression) had a statistically significant reduction in scores in yoga group at 3 months. The EuroQOL scores increased significantly in the Yoga group at both 1 month and 3-month time points, indicating that QOL improved more in patients receiving IAYT. The GEE was used to find the overall change in $p$-value over time between the groups. All parameters had a significant $p$-value. $(<0.001)$ except for PDQ $(p=0.195)$. The adjusted mean difference $(95 \% \mathrm{Cl})$ with effect size between Yoga and Control group at 1 month and 3 months for above mentioned secondary outcomes are provided in Table 3. 
Table 3

Adjusted mean difference $(95 \% \mathrm{Cl})$ between Yoga and Control and at 3 months with an effect size.

\begin{tabular}{|c|c|c|c|c|c|}
\hline \multirow[t]{2}{*}{ Variable } & Yoga $(n=14)$ & Control(n=15) & \multirow{2}{*}{$\begin{array}{l}\text { Adjusted mean } \\
\text { difference }(95 \% \mathrm{Cl})\end{array}$} & \multirow{2}{*}{$\begin{array}{l}\text { Effect } \\
\text { size }\end{array}$} & \multirow[t]{2}{*}{$P$ value } \\
\hline & Mean \pm SD & Mean \pm SD & & & \\
\hline \multicolumn{6}{|c|}{ Adjusted mean difference at 3 months } \\
\hline DN4\# & $1.07 \pm 1.07$ & $2.93 \pm 1.71$ & 1.86 & 1.32 & $0.003^{1}$ \\
\hline PDQ\# & $5.14 \pm 3.63$ & $7.93 \pm 3.59$ & 2.79 & 0.79 & $0.04^{1}$ \\
\hline PCS\# & $\begin{array}{l}15.93 \\
\pm 10.16\end{array}$ & $27.47 \pm 8.37$ & 11.54 & 1.27 & $0.003^{1}$ \\
\hline EuroQOL* & $0.65 \pm 0.10$ & $0.41 \pm 0.23$ & 0.24 & 1.33 & $<0.001^{1}$ \\
\hline HADS (ANXIETY)\# & $6.36 \pm 3.71$ & $9.47 \pm 3.31$ & 3.11 & 0.9 & $0.02^{1}$ \\
\hline HADS(DEPRESSION)\# & $5.43 \pm 3.32$ & $7.00 \pm 3.02$ & 1.57 & 0.69 & 0.195 \\
\hline TAMPA\# & $\begin{array}{l}33.71 \pm \\
5.33\end{array}$ & $44.53 \pm 5.25$ & 10.82 & 2.08 & $<0.001^{1}$ \\
\hline \multicolumn{6}{|c|}{ Adjusted mean difference at 3 months. } \\
\hline DN4\# & $0.57 \pm 0.65$ & $2.20 \pm 1.66$ & 1.63 & 1.3 & $0.007^{1}$ \\
\hline PDQ\# & $2.71 \pm 3.20$ & $6.40 \pm 4.15$ & 3.69 & 1.01 & $0.005^{1}$ \\
\hline PCS\# & $\begin{array}{l}11.86 \\
\pm 11.92\end{array}$ & $24.80 \pm 10.07$ & 12.94 & 1.27 & $0.003^{1}$ \\
\hline EuroQOL* & $0.76 \pm 0.20$ & $0.46 \pm 0.23$ & 0.30 & 1.43 & $0.001^{1}$ \\
\hline HADS (ANXIETY)\# & $5.07 \pm 3.73$ & $9.13 \pm 3.36$ & 4.07 & 1.17 & $0.005^{1}$ \\
\hline HADS (DEPRESSION\# & $3.71 \pm 3.12$ & $6.87 \pm 3.18$ & 3.16 & 1.02 & $0.012^{1}$ \\
\hline TAMPA\# & $\begin{array}{l}30.64 \pm \\
6.67\end{array}$ & $42.87 \pm 6.09$ & 12.23 & 1.95 & $0.001^{1}$ \\
\hline \multicolumn{6}{|c|}{$\begin{array}{l}\text { Values represented as mean } \pm \text { SD, } 1 \text { - significant } p \text {-value }<0.05 \text {, \# - reduction in score indicates better } \\
\text { outcome, *increase in score indicates the improved quality of life. Wilcoxon-Mann-Whitney test was } \\
\text { used for DN4, PDQ, EuroQOL, PCS, TAMPA for intergroup comparison at } 1 \text { and } 3 \text { months. T-test was } \\
\text { used for HADS (anxiety and depression) for intergroup comparison at } 1 \text { and } 3 \text { months. }\end{array}$} \\
\hline
\end{tabular}

\section{Drug therapy:}

Patients in both the groups were either on pregabalin or amitriptyline as monotherapy or a combination of both the drugs as per treatment protocol. Baseline drug therapies were comparable in both groups. The 
baseline mean dosage of pregabalin in yoga group was $78.57 \pm 67.12 \mathrm{mg} /$ day a significant reduction in dosage of pregabalin was noted over time, at 3 months the dosage reduced to $30.57 \pm 13.36 \mathrm{mg} /$ day $(p=0.001)$. The baseline pregabalin dosage in control group was $85.00 \pm 66.68 \mathrm{mg} /$ day with dosage at three months $95.00 \pm 87.73$ ( $p=0.819)$. Inter-group comparison revealed a significant dose reduction in the Yoga group compared to the Control group overtimes $(p=0.001)$. For the doses of amitriptyline, a significant reduction $(p=0.008)$ from baseline $(9.29 \pm 2.67)$ to three months $(5.71 \pm 7.03)$ was noted only in yoga group. No significant dose reduction of amitriptyline was observed in control group. On intergroup comparison no significant difference in dose of amitriptyline was seen in both the groups over time. For the combined pregabalin plus amitriptyline, a significant dose reduction was reported from baseline (87.86 \pm 50.44$)$ both at $1(49.64 \pm 50.44)$ and 3 months $(10.00 \pm 15.93)$ in the Yoga group $(p=0.001)$. No significant reduction was observed in control group. The Yoga group had a significant reduction in comparison to the control group $(p=0.001)$.

A total of 6 out of 14 (42.9\%) patients in the yoga group stopped medication at three months due to improvement. Four patients were switched from dual to monotherapy at 1 month in the Yoga group. In the Control group, none of the patients stopped medication at three months. Only two patients in control group switched from dual therapy to monotherapy at three months. On comparative analysis, a significant number of patients stopped medication at 3 months in the Yoga group comparison to the Control group. $(p=0.008)$.

\section{Biomarker's analysis}

We analyzed 21 samples for assessment of TNF-a in the serum of CLBP patients. Eight cases were excluded due to errors while processing samples. The within the group analysis of subsequent follow-up samples ( 1 month and 3 months) showed significant reduction in TNF-a levels of yoga group $(p=0.004)$ as compared to control group $(p=0.002)$ GEE analysis revealed comparable TNF-a levels compared over time $(p=0.77)$. A total of 24 participants samples were processed for levels of beta-endorphins. Five cases were excluded due to sample processing errors. We found that the beta-endorphins levels were comparable at the base line $(0.02 \pm 0.01) \mathrm{pg} / \mathrm{mL}$ and showed non-significant increase in yoga group to $(0.03 \pm 0.01) \mathrm{pg} / \mathrm{mL}$ at one month and 3 month as compared to the control group $(0.02 \pm 0.01) \mathrm{pg} / \mathrm{mL}$.

A total of 22 samples were processed for estimation of salivary CGRP. Seven patients were excluded due to sample processing error. We found that the CGRP levels differed significantly at the baseline $(p=0.001)$ and also at 1 month follow-up $(p=0.006)$ between yoga $(5.92 \pm 2.74) \mathrm{pg} / \mathrm{mL}$ and control groups $(8.42 \pm$ 1.67) $\mathrm{pg} / \mathrm{mL}$.

\section{Discussion}

The result of our study revealed significant reduction in VNRS in the yoga group in comparison to the control group over time with medium to large effect. Also, significant improvement in MODI was observed in the yoga group. A moderate to large effect was seen both at one and three months (effect size $=1.33$ and 1.35 respectively) with an overall $p$-value $=0.001$. Additionally, we observed significant improvement 
in all secondary outcomes both at 1 month and 3 months in the Yoga group compared to the Control group

Few studies have evaluated the role of yoga therapy for management of CLBP patients. Saper et al revealed a significant reduction in pain after the 12 weeks of yoga intervention, irrespective of the number of yoga classes attended.[35] A systemic review by Chang et al reported that Yoga reduces pain in CLBP patients.[36] Another comprehensive review reported an analysis of eight randomized controlled trials.[37] The results indicated that the overall post-treatment effect size of yoga on pain was $0.623(95 \% \mathrm{Cl} 0.377$ to 0.868 ) indicating a medium to large effect which reduced with time. We observed comparable results in the present study. At 1 month the effect size was 0.83 ( $95 \% \mathrm{Cl}: 0.15$ to 2.55 ) indicating medium to large effect. On following at 3 months the reported effect size was reduced to 0.48 (95\% Cl:0.61 to 2.87).

Two of the meta-analysis and a systematic review, recommending yoga as a beneficial adjuvant approach for CLBP reported moderate effect sizes on disability. [10, 36-39] Holtzman et al noted that the post-yoga effect for functional disability was in the moderate-to-large range, $d=0.645$.[37] We noted similar moderate to large effect at one and three months (effect size $=1.33$ and 1.35 respectively) in our study Yoga group. Our results are also in strong approval with the existing guideline recommendation on nonpharmacological therapies, as the first-line treatment method for CLBP patients. [36-39]

Neuropathic pain was found to be considerably reduced with yoga in our trial. The present study thus provides evidence that yoga therapy reduces pain even of neuropathic origin. Comparative data with other studies in respect to PDQ and DN4 is not available at present, but a recent study validates that Yoga is a cost-effective approach for the management of neuropathic pain. [40,41]

CLBP is a complex condition that affects all aspect of life. This results in economic burden, reduced work productivity and affects the quality of life(QOL).[42]Yoga Studies for CLBP patients conducted so far have limited data on the measures of QOL, mental health conditions (depression, anxiety, etc), and other psychological impacts.[10,43-45] Tekuret al demonstrated the usefulness of IAYT in improving QOL within the CLBP nursing populations.[15] They observed a significant improvement in all the four domains of WHOQOL-BREF in the yoga-based lifestyle module as compared to the physical exercisebased lifestyle module.[15] The present study reciprocates similar results. A significant increase in EuroQol score over time was noted in Yoga group compared to Control group.

We found depression score using HADS was lower in the yoga group at both time points with significant reduction at 3 months. This is consistent with the past research, where in Yoga, CBT, and MBSR have reported to be substantially effective in reducing depression and anxiety in CLBP patients. [39, 43-45] Anxiety was significantly reduced in yoga group, both at $1(p=0.02)$ and 3 months $(p=0.005)$ compared to control group. The additional benefits of yoga therapy like social connectedness and spirituality as highlighted in recent reports, enhanced its primary effects of decreasing disability and promoting mental well-being.[46] 
We studied both kinesiophobia and the catastrophic effects of pain by TSK and PCS.[47] Our study reported a significant reduction for both parameters in the Yoga group compared to the control group at all time points. This was in contrast to the study of William et al who did not report a significant reduction in kinesiophobia after lyenger yoga therapy up to 16 weeks.[48] William et al explained the lack of treatment effect on the psychological and behavioral subscales by the following facts.[48] Firstly, the study was not adequately powered. Secondly, more time may be required to change long-held negative cognitions and beliefs about CLBP such as movement-related pain. The lyengar technique of YOGA involves a large number of difficult standing postures that require repeated practice to obtain correct pelvic alignment thus diminished the efficacy of the intervention. Lastly, that the impact of the yoga intervention would have been greater with a more experienced instructor. In contrast, the present study used the IAYT and not the IYENGER yoga technique and ensured a more experienced instructor which helped patients in easily understanding the techniques. These differences can be attributed to a significant effect in both TSK and PCS.

A noteworthy observation in our study is significant reduction in pain medication dosage with some patients becoming off medications at 3 months. We noted that $42 \%$ of patients become off medication in the yoga group and a significant in dosage was observed in comparison to the control group $(p=0.008)$. These findings correlate with the results of randomized controlled trials.[48] William et al reported $88 \%$ of the patients in the yoga group having a reduction in medication with significant reduction in Yoga group compared to control group.[48] This finding further adds clinical relevance to using yoga as adjuvant therapy in patients with CLBP.

We wanted to see efficacy of Yoga as an adjuvant to prescribed medical treatment in CLBP patients at molecular level. For this we estimated salivary CGRP along with serum TNF- $a$ and beta-endorphin in both the groups. Neuro-inflammatory processes affect inflammatory mediators such as TNF-a in the plasma. The elevated levels are linked to elevated pain intensity which plays an important role in CLBP patients $[49,50]$. In the present study TNF-alpha, reduced significantly as the pain intensity reduced over time in both groups. But the comparative analysis did not have a statistically significant value. Our results align with a study of overweight people with chronic inflammatory conditions where reduced TNFa levels were found in those who practiced yoga,[50] but in this study, there was no comparative control group and the study was a short-term follow-up of 12 weeks. The reduction in TNF alpha cannot be attributed to YOGA due to multiple confounders. The TNF-a levels are altered by amitriptyline intake [51] which could act as confounder, hence we found significant decrease in the TNF-a levels in both the groups.

We also studied levels of beta-endorphins and salivary CGRP. The beta-endorphins are the most extensively researched and beta-endorphin level may serve as a surrogate marker for treatment response of patients with CLBP.[30] In patients with CLBP, beta-endorphin levels have been reported suppressed stably and not affected by a placebo effect. [31, 52] We found that beta-endorphins showed an increasing trend in Yoga group as compared to control group although it was not statistically significant. Research indicates that CGRP might have a pro-inflammatory role in the peripheral nervous system by releasing 
pro-nociceptive substances, and they also found the relationship between measured CGRP levels and neuropathic pain.[33] CGRP is an abundant neuropeptides in nervous tissue and also present in saliva and plays a significant role in the pathophysiology of chronic pain.[34] Salivary CGRP shows a significant increase in saliva and plasma concentration of CGRP during headache episodes and migraine, especially during active pain periods.[53]

The non-significant change in the beta-endorphins can be attributed to the confounders like psychological state, pain intensity, and medications. Amitriptyline which being an anti-depressant is known to affect the level of this biomarker. [54] Similarly, Pregabalin is known to modulate CGRP levels which might be implicated for non-significant change in its serum levels in both the groups.[55] Moreover TNF-a is known to control CGRP expression [56] and as two these molecules are synergistically lowered in our study suggesting their involvement in some common pain related pathway. TNF-a, beta-endorphins and CGRP interact with various other genes. In inflammatory conditions like acute pancreatitis TNF- $a$, interleukins 3,6 are downregulated and MAPK, NF-KB, and STAT3 signaling is inhibited in CGRP dependent manner.[57] Hence, we propose that further studies must be carried out after adjusting the confounders like age, gender, comorbidities and life style habits, with larger sample size will also add to the strength of the study.

No adverse events were reported during the Yoga Intervention.

\section{Limitation:}

One of the limitations of this study is small sample size with pilot feasibility design. However, post hoc power analysis revealed power of $88 \%$ with a MICD 2 and SD of 2.2, a error of 0.05 and sample size of 29. Another limitation is that the patients and physician could not be blinded because of design of study. However, the bias was taken care by keeping the follow up researcher and statistician blinded to the group allocated and keeping the study cases in between the routine clinical cases. Another limitation is short follow up of 3 months, this was primarily due to logistic reasons with many patients attending the pain clinic from far of remote places. Future randomized clinical trial including a larger patient population with longer follow up are warranted.

\section{Conclusion}

In Conclusion, IAYT reinforces the possibilities of being a substantial adjunct intervention to reduce pain symptoms and pain-associated disability in adult patients with CLBP. It seems to have a positive effect on depression and anxiety and even benefitting the overall QOL, with maintaining its neutral effect on the Pain biomarkers.

\section{Declarations}

Acknowledgements

Page 15/23 
Priya Battu (Laboratory Experiments and manuscript editing), Sheetal Jindal (Blood Sample collection and Lab experiments), Prashant (Blood Sample collection Conducting few Yoga Sessions \& Brochure Contribution) Raman Nautiyal (statistician)

\section{Competing interest}

The authors declare no competing interest

\section{Funding Sources}

None

\section{Author contribution}

S.P -main project researchers conducted cases, follow up and drafting of manuscript, D.D- Drafting results , data interpretation and manuscript writing, B.G - corresponding author, initiating research , patient assessment, manuscript drafting and final formatting. D.M- correcting formatting manuscript, K.M - formatting study project and manuscript formatting, K.K - manuscript formatting, sample collection. A.A-Biomarkers assessment, results, conducting tests and manuscript formatting.

\section{References}

1. Buchbinder R, van Tulder M, Öberg B, Costa LM, Woolf A, Schoene M, et al. Low back pain: a call for action. The Lancet. 391, 2384-8. (2018)

2. Vos T, Allen C, Arora M, Barber RM, Brown A, Carter A, et al. GBD 2015, Global, regional, and national incidence, prevalence, and years lived with disability for 310 diseases and injuries, 1990-2015: a systematic analysis for the Global Burden of Disease Study 2015. The Lancet. 388, 1545-602(2016)

3. James SL, Abate D, Abate KH, Abay SM, Abbafati C, Abbasi N, et al. GBD 2017, Global, regional, and national incidence, prevalence, and years lived with disability for 354 Diseases and Injuries for 195 countries and territories, 1990-2017: A systematic analysis for the Global Burden of Disease Study 2017. The Lancet, $1789-858$ (2018)

4. Bansal D, Asrar MM, Ghai B, Pushpendra D. Prevalence and Impact of Low Bac Pain in a CommunityBased Population in Northern India. Pain Physician. 23, 389-398(2020)

5. Asrar MM, Ghai B, Pushpendra D, Bansal D. Psychosocial morbidity profile in a community-based sample of low back pain patients. Sci Rep. 11, 2610(2021)

6. Day MA, Ward LC, Ehde DM, Thorn BE, Burns J, Barnier A, et al. A Pilot Randomized Controlled Trial Comparing Mindfulness Meditation, Cognitive Therapy, and Mindfulness-Based Cognitive Therapy for Chronic Low Back Pain. Pain Medicine (United States). 20, 2134-48(2019).

7. Saper RB, Lemaster C, Delitto A, Sherman KJ, Herman PM, Sadikova E, et al. Yoga, physical therapy, or education for chronic low back pain: A randomized noninferiority trial. Annals of Internal Medicine. 167, 85-94(2017). 
8. Cherkin DC, Sherman KJ, Balderson BH, Cook AJ, Anderson ML, Hawkes RJ, et al. Effect of mindfulness-based stress reduction vs cognitive behavioral therapy or usual care on back pain and functional limitations in adults with chronic low back pain: A randomized clinical trial. JAMA Journal ontrol trof the American Medical Association. 315, 1240-9(2016).

9. Tilbrook HE, Cliaox H, Hewitt CE, Kang'ombe AR, Chaung LH, Jayakody S et al . Yoga for Chronic Low Back Pain.: a randomized control trial. Annals of Internal Medicine. 155, 569-78 (2011).

10. Wieland L, Skoetz N, Pilkington K, Vempati R, D Adamo CR, Berman BM. Yoga treatment for chronic non-specific low back pain. Cochrane Database of Systematic Reviews. 12, 1(2017).

11. Qaseem A, Wilt TJ, McLean RM, Forciea MA. Noninvasive treatments for acute, subacute, and chronic low back pain: A clinical practice guideline from the American College of Physicians. Annals of Internal Medicine.166, 514-30(2017).

12. Goyal M, Haythornthwaite JA. Is It Time to Make Mind-Body Approaches Available for Chronic Low Back Pain? Data Sharing An Ethical and Scientific Imperative. JAMA - Journal of the American Medical Association. 315, 1236-7(2016).

13. Patil N, Nagaratna R, Tekur P, Manohar P, Bhargav H, Patil D. A randomized trial comparing effect of yoga and exercises on quality of life in among nursing population with chronic low back pain. International Journal of Yoga. 11, 208 (2018)

14. Patil NJ, Nagarathna R, Tekur P, Patil DN, Nagendra HR, Subramanya P. First phase : Designing of integrated yoga therapy module for chronic low back pain Second phase : Validation of integrated yoga therapy module for chronic low back pain Third phase : Feasibility study Results : Feasibility study. International Journal of Yoga. 2, 26-8 (2015).

15. Tekur P, Chametcha S, Hongasandra R, Raghuram N. Effect of yoga on quality of life of CLBP patients: A randomized control study. International Journal of Yoga. 3, 10 (2010).

16. Sherman KJ, Wellman RD, Cook AJ, Cherkin DC, Ceballos RM. Mediators of Yoga and Stretching for Chronic Low Back Pain. Evid Based Complementary and Alternat Med.130818(2013). doi: $10.1155 / 2013 / 130818$.

17. Lee M, Moon W, Kim J. Effect of Yoga on Pain, Brain-Derived Neurotrophic Factor, and Serotonin in Premenopausal Women with Chronic Low Back Pain. Evid Based Complementary and Alternat Med. 203173 (2014). doi: 10.1155/2014/203173

18. Cho HK, Moon W, Kim J. Effects of yoga on stress and inflammatory factors in patients with chronic low back pain: A non-randomized controlled study. European Journal of Integrative Medicine [Internet]. 7, 118-23 (2015)

19. Ghai B, Gudala K, Asrar M, Chanana N, Kanukula R, Bansal D. Development, validation and evaluation of a novel self-instructional module in patients with chronic non-specific low back pain. Indian Journal of Anaesthesia. 64, 299-305 (2020).

20. H. Breivik, P. C. Borchgrevink SMA. Assessment of pain. British Journal of Anaesthesia. 101, 10-8 (2008). 
21. Fritz JM, Irrgang JJ. A comparison of a modified Oswestry Low Back Pain Disability Questionnaire and the Quebec Back Pain Disability Scale. Physical therapy [Internet]. 81, 776-88(2001).

22. Bouhassira D, Attal N, Alchaar H, Boureau F, Brochet B, Bruxelle J, et al. Comparison of pain syndromes associated with nervous or somatic lesions and development of a new neuropathic pain diagnostic questionnaire (DN4). Pain. 114, 29-36 (2005).

23. Masahiko Sumitani HA, Matsubayashi Y, Tsuchida R, Oshima Y, Takeshita K, Yamada Y. Validation of Pain Severity Assessment using the PainDETECT Questionnaire. International Journal of Anesthesiology \& Pain Medicine. 03, 1-5 (2017).

24. Bansal D, Gudala K, Lavudiya S, Ghai B, Arora P. Translation, adaptation, and validation of hindi version of the pain catastrophizing scale in patients with chronic low back pain for use in India. Pain Medicine (United States). 17, 1848-58 (2016).

25. Soer R, Reneman MF, Speijer BLGN, Coppes MH, Vroomen PCAJ. Clinimetric properties of the EuroQol-5D in patients with chronic low back pain. Spine Journal. 12, 1035-9 (2012).

26. Turk DC, Dworkin RH, Trudeau JJ, Benson C, Biondi DM, Katz NP, et al. Validation of the Hospital Anxiety and Depression Scale in Patients with Acute Low Back Pain. Journal of Pain. 16, 1012-21 (2015).

27. Swinkels-Meewisse EJCM, Swinkels RAHM, Verbeek ALM, Vlaeyen JWS, Oostendorp RAB. Psychometric properties of the Tampa Scale for kinesiophobia and the fear-avoidance beliefs questionnaire in acute low back pain. Man Ther. 8, 29-36 (2003).

28. Rampakakis E, Ste-Marie PA, Sampalis JS, Karellis A, Shir Y, Fitzcharles MA. Real-life assessment of the validity of patient global impression of change in fibromyalgia. RMD Open. 1, 1-6 (2015).

29. Ferguson L, Scheman J. Patient global impression of change scores within the context of a chronic pain rehabilitation program. The Journal of Pain. 10, 73 (2009).

30. Choi HY, Lee $\mathrm{CH}$. Can beta-endorphin be used as a biomarker for chronic low back pain? A metaanalysis of randomized controlled trials. Pain Medicine (United States). 20, 28-36 (2018).

31. Takahashi M, Yoshida A, Yamanaka H, Furuyama Y, Horinouchi T, Kato M, et al. Lower $\beta$-endorphin content of peripheral blood mononuclear cells in patients with complex regional pain syndrome. $J$ Back Musculoskelet Rehabil. 15, 31-6 (2000)

32. Klyne DM, Barbe MF, Hodges PW. Systemic inflammatory profiles and their relationships with demographic, behavioural and clinical features in acute low back pain. Brain, Behavior, and Immunity. 60, 84-92(2017).

33. Schou WS, Ashina S, Amin FM, Goadsby PJ, Ashina M. Calcitonin gene-related peptide and pain: a systematic review. Journal of Headache and Pain. 18, 34 (2017).

34. Fischer HP, Eich W, Russell IJ. A possible role for saliva as a diagnostic fluid in patients with chronic pain. Seminars in Arthritis and Rheumatism. 27, 348-59 (1998).

35. Saper RB, Boah AR, Keosaian J, Cerrada C, Weinberg J, Sherman KJ. Comparing Once-versus TwiceWeekly Yoga Classes for Chronic Low Back Pain in Predominantly Low Income Minorities: A Randomized Dosing Trial. Evidence-Based Complementary and Alternative Medicine. 1-13 (2013). 
36. Chang DG, Holt JA, Sklar M, GroessI EJ, Diego S, Diego S, et al. U.S. Department of Veterans Affairs review of the literature. J OrthopRheumatol. 3, 1-8(2016).

37. Holtzman S, Beggs RT. Yoga for chronic low back pain: a meta-analysis of randomized controlled trials. Pain Research and Management. 18, 267-72 (2013)

38. Holger Cramer, MSc, RomyLauche, PhD, Heidemarie Haller, MSc, and Gustav Dobos M, Objectives: A Systematic Review and Meta-analysis of Yoga for. Clin J Pain. 5, 1-11(2013)

39. Lee H, Hübscher M, Moseley GL, Kamper SJ, Traeger AC, Mansell G, et al. How does pain lead to disability? A systematic review and meta-analysis of mediation studies in people with back and neck pain. Pain. $156,988-97(2015)$.

40. Chou R, Deyo R, Friedly J, Skelly A, Hashimoto R, Weimer M et al. Nonpharmacologic Therapies for Acute and Chronic Low Back Pain: A Review of the Evidence for an American Pain Society/American College of Physicians Clinical Practice Guideline. Ann Intern Med. 166, 493-500(2007).

41. Fields HL. Should we be reluctant to prescribe opioids for chronic non-malignant pain?. Pain. 129, 233-4 (2007).

42. Hartvigsen J, Hancock MJ, Kongsted A, Louw Q, Ferreira ML, Genevay S, et al. What low back pain is and why we need to pay attention. The Lancet. 391, 2356-67(2018).

43. Cramer $\mathrm{H}$, Haller $\mathrm{H}$, Lauche R, Dobos G. Mindfulness-based stress reduction for low back pain. A systematic review. BMC Complement Altern Med. 12, 162 (2012).

44. Trocoli TO, Botelho R v. Prevalence of anxiety, depression and kinesiophobia in patients with low back pain and their association with the symptoms of low back spinal pain. RevistaBrasileira de Reumatologia. 56, 330-6(2016).

45. Kuvačić G, Fratini P, Padulo J, Antonio DI, de Giorgio A. Effectiveness of yoga and educational intervention on disability, anxiety, depression, and pain in people with CLBP: A randomized controlled trial. Complementary Therapies in Clinical Practice. 31, 262 (2018)

46. Vallerand AH, Cosler P, Henningfield JE, Galassini P. Pain management stratigies and lessons fron the military: A narrative review. Pain Res Manag. 20, 261-68(2015)

47. Crombez G, Eccleston C, van Damme S, Vlaeyen JW, Karoly P. Fear-Avoidance Model of Chronic Pain: the Next Generation. Clin J Pain. 28 , 475-83 (2012).

48. Williams K, Abildso C, Steinberg L, et al. Evaluation of the effectiveness and efficacy of lyengar yoga therapy on chronic low back pain. Spine (Phila Pa 1976). 34, 2066-2076 (2009).

49. Jasim H, Carlsson A, Hedenberg-Magnusson B, Ghafouri B, Ernberg M. Saliva as a medium to detect and measure biomarkers related to pain. Scientific Reports. 8, 1-9(2018).

50. Wang H, Schiltenwolf M, Buchner M. The role of TNF-a in patients with chronic low back pain - A prospective comparative longitudinal study. Clinical Journal of Pain. 24, 273 (2008)

51. Khan AN, Jacobsen HE, Khan J, Filippi CG, Levine M, Lehman RA, et al. Inflammatory biomarkers of low back pain and disc degeneration: a review. Annals of the New York Academy of Sciences. 1410, 68-84 (2017). 
52. Yadav RK, Magan D, Mehta N, Sharma R, Mahapatra SC. Efficacy of a short-term yoga-based lifestyle intervention in reducing stress and inflammation: Preliminary results. Journal of Alternative and Complementary Medicine. 18, 662-7 (2012).

53. van Dongen RM, Zielman R, Noga M, Dekkers OM, Hankemeier T, van den Maagdenberg AMJM, et al. Migraine biomarkers in cerebrospinal fluid: A systematic review and meta-analysis. Cephalalgia. 27, 49-63 (2017).

54. Jadrić R, Kiseljaković E, Hasić S, Winterhalter-Jadrić M. ß-endorphins as Possible Markers for Therapeutic Drug Monitoring. Bosn J Basic Med Sci. 7, 11-14 (2007).

55. Fehrenbacher JC, Taylor $C P$, Vasko MR. Pregabalin and gabapentin reduce release of substance $P$ and CGRP from rat spinal tissues only after inflammation or activation of protein kinase C. Pain. 105, 133-41 (2003).

56. Hinze-Selch D, Schuld A, Kraus T, Kühn M, Uhr M, Haack M, Pollmächer T. Effects of antidepressants on weight and on the plasma levels of leptin, TNF- $a$ and soluble TNF receptors: a longitudinal study in patients treated with amitriptyline or paroxetine. Neuropsychopharmacology. 23, 13-9(2000).

57. Huang $H$, Wang $M$, Guo Z, Wu D, Wang $H$, Jia $Y$ et al. Rutaecarpine alleviates acute pancreatitis in mice and AR42J cells by suppressing the MAPK and NF-KB signaling pathways via calcitonin generelated peptide. Phytother Res. 35, 6472-85 (2021)

\section{Figures}




\section{ORIENTATION \&} CONDUCTION

\section{FOLLOW UP - 1} MONTH

ANALYSIS

\section{FOLLOW UP - 3 MONTH}

\section{Figure 1}

Consort flow diagram 


\section{Change in VNRS Over Time}

$$
\text { Group - Yoga - Control }
$$

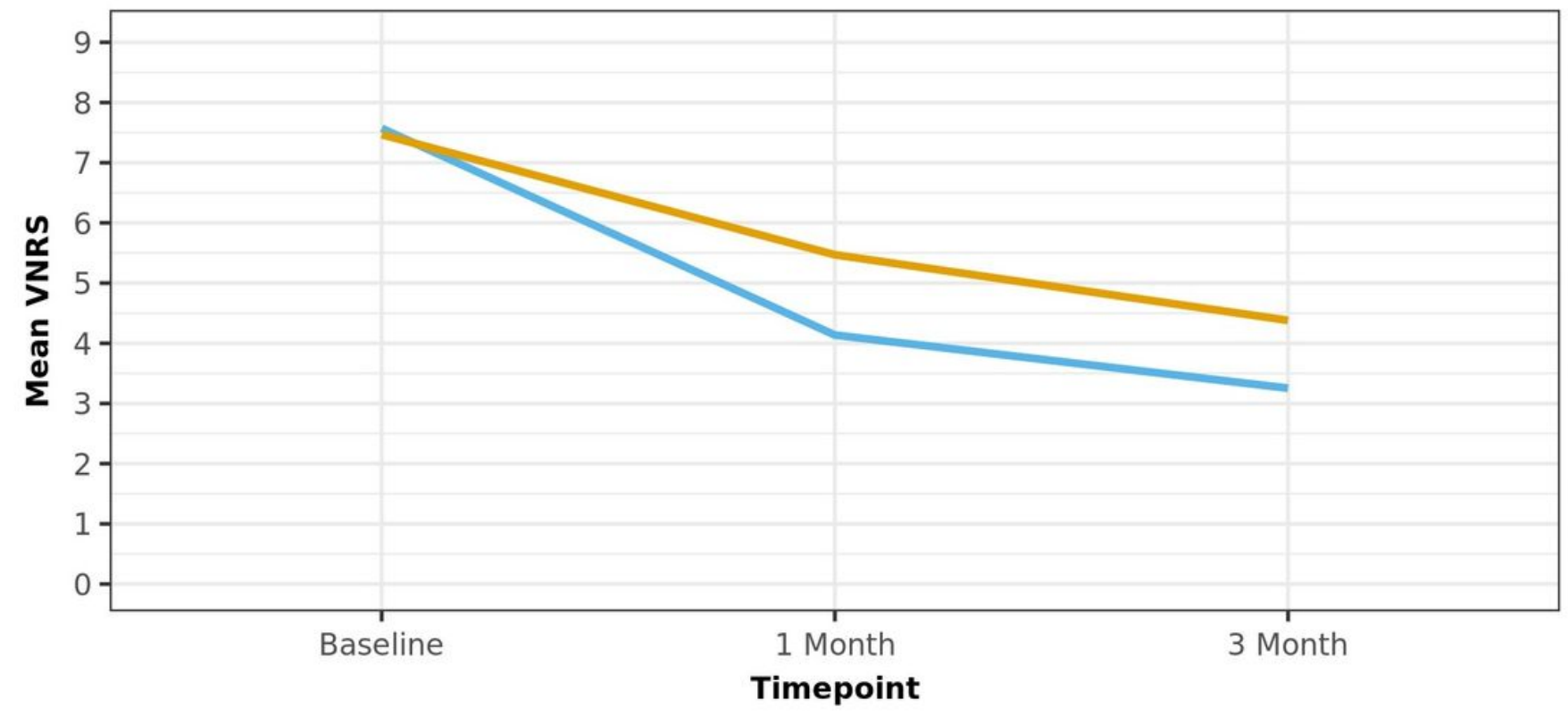

\section{Figure 2}

Line diagram showing the change in VNRS over time in both groups. 


\section{Change in MODQ Over Time}

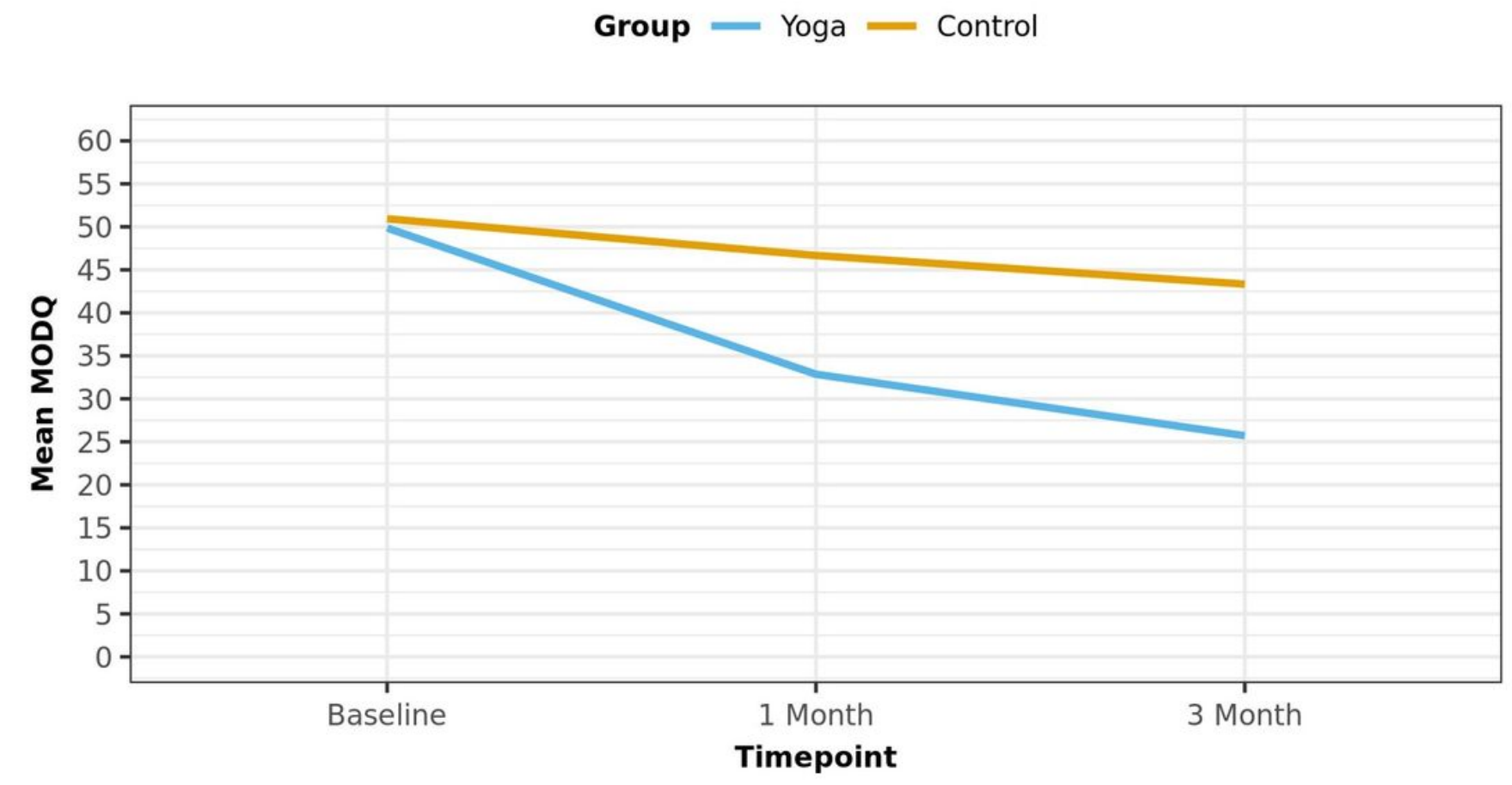

Figure 3

Line diagram showing the change in MODQ over time in both groups. 